\title{
Optical Injection Locking for Carrier Phase Recovery and Regeneration
}

\author{
Radan Slavík ${ }^{1} *$ Zhixin Liu $^{1,2}$, and David J. Richardson ${ }^{1}$ \\ 1. Optoelectronics Research Centre, University of Southampton, Southampton, SO17 1BJ, UK \\ 2. Now with Optical Networks Group, Dept. of Electronic \& Electrical Engineering, University College London, London, U.K. \\ *r.slavik@soton.ac.uk
}

\begin{abstract}
We review various scenarios for using optical injection locking for phase synchronization of signals to a local oscillator. We concentrate on the principle of operation and key properties needed. (C2017 Optical Society of America

OCIS codes: (060.2320) Fiber optics amplifiers and oscillators, (140.3520) Laser, injection-locked.
\end{abstract}

\section{Introduction}

Coherent optical detection was initially researched due to its ability to detect very weak signals without suffering from thermal noise in the photodetector. However, the difficulty of phase-synchronizing the optical local oscillator (CW laser) with incoming data proved to be a significant technical challenge, preventing adoption of coherent detection by the industry. The advantages of coherent communications, however, go well beyond the capability of overcoming thermal noise limitations (which was addressed in the meantime by using Erbium-doped fiber amplifiers, EDFAs). Some of these advantages include enabling higher spectral efficiency and the ability to compensate for linear impairments. This is why coherent optical communications was resurrected in more recent times, although the original (challenging) approach of phase locking of the local oscillator with the data was replaced by 'software' based phase synchronization using fast electronic Digital Signal Processing (DSP).

DSP offers unparalleled possibilities in the manipulation of signals. However, it can only be implemented after photodetection and digitization (analog-to-digital conversion, ADC). This may be undesirable (e.g., in mid-span regeneration, in which the signal is further transmitted on an optical carrier) and is often simply not possible in many scenarios (e.g., in quantum optics). The use of very complex DSP (especially in conjunction with Forward Error Correction, which is almost always used in coherent communications) is also associated with increased latency, which is highly-undesirable in many applications such as high-frequency trading and 5G mobile communications [1]. In these fields, phase locking of the local oscillator to the incoming data could be beneficial not only for coherent detection, but also for processing of the signal directly in the optical domain, which is commonly referred to as Optical Signal Processing.

Phase-locking of a local oscillator with incoming data can be done by various means. One of the two main challenges is the relatively high phase noise (spectral purity, sometimes characterized in terms of the laser linewidth) of the free-running lasers that serve as local oscillators. To phase-lock the incoming signal with the local oscillator (CW laser) using traditional electronic-based phase-locked loops (PLL) requires a relatively large bandwidth PLL. Such PLLs are becoming available, making this approach appealing [2]. An alternative to the electronic phaselocked loop is an effect called Optical Injection Locking (OIL). In OIL, an optical signal (Master signal) is injected into a laser (Slave laser), which can under certain conditions start to follow the phase of the Master signal.

The other main challenge in phase synchronization of a CW laser with an incoming optical signal is the fact that no optical carrier signal is actually transmitted in most popular modulation formats (e.g. QPSK, 16 QAM and higher). This is highly-advantageous the transmission, as carrier power often triggers unwanted non-linear effects. However, the absence of the signal carrier poses a significant challenge for phase synchronization between the local oscillator and the optical data signal.

Here, we review the topic of phase synchronization of CW lasers (local oscillators) to incoming data using OIL and briefly discuss different scenarios of carrier generation from signals that do not intrinsically contain the carrier signal. We show various scenarios in which this is used, including for homodyne coherent detection, optical regeneration using all-optical methods such as Phase Sensitive Amplification, and the extraction of tones from Optical Frequency Combs.

\section{Optical Injection Locking (OIL)}

OIL refers to the phenomenon under which the Slave laser (subject to the external injection of a Master signal) is phase and frequency locked to the Master signal. OIL takes place only if the difference in frequency between the 
Master signal and free running Slave laser $\left(f_{\text {Master }}-f_{\text {Slave }}^{\text {FreeRuning }}\right)$ is within a certain bandwidth known as the locking bandwidth $\left(\mathrm{BW}_{\text {OIL }}\right)$. The locking bandwidth is proportional to the square root of the injection ratio $\rho_{\text {OIL }}[3]$ :

$$
\mathrm{BW}_{\text {OIL }} \approx \sqrt{\rho_{\text {OIL }}}=\sqrt{\frac{P_{\text {Master }}}{P_{\text {Slave }}}},
$$

where $P_{\text {Master }}$ is the optical power of the Master injected into the Slave laser and $P_{\text {Slave }}$ is the optical power of the free running Slave laser.

Roughly speaking, OIL is usually operated within one of the following three regimes:
a) Large OIL ratio $\left(\rho_{\text {OIL }}>-6 d B\right)$
b) Low OIL ratio ( $-6 d B>\rho_{\text {OIL }}>-30 d B$ ); an example of a measured OIL range is shown in Fig. 1a
c) Ultra-low OIL ratio $\left(\rho_{O I L}<-30 \mathrm{~dB}\right)$

\section{$\underline{2.1 \text { Large OIL ratio }}$}

A large OIL ratio strongly influences the Slave laser dynamics, allowing, for example, modulation bandwidth enhancement [4]. In general, Eq. (1) is not valid in this regime and is beyond the scope of this paper.

\subsection{Low OIL ratio}

The low OIL ratio regime is the one most commonly used for phase synchronization of a Slave laser to a Master signal. In many applications, phase synchronization over as large a bandwidth as possible is required. An example is Slave laser linewidth narrowing using a narrow-linewidth Master laser [5], or extraction of a single tone of an Optical Frequency Comb (which plays the role of the Master signal [6]), as shown in Fig. 1b. An additional feature of the low OIL ratio regime is that the Slave laser power is relatively insensitive to fluctuations in the Master signal, giving rise to amplitude regeneration of the injected signal. This effect can be used for Master signal RIN (Relative Intensity Noise) reduction, or for amplitude regeneration of constant-amplitude modulated signals (e.g., BPSK/QPSK) [7].

In all the afore-mentioned 'Low OIL ratio' regime applications, the Master signal is a low-phase noise (linewidth) signal and we want to 'copy' its low-noise phase properties onto the Slave laser - generally to either (i) improve the amplitude noise properties, (ii) amplify the (weak) Master signal, or (iii) 'clone' the Master laser (e.g., for "squeezed vacuum" (vacuum with quantum-limited noise unevenly distributed between amplitude and phase) experiments [8]. The situation is different in optical communications, where the optical signal is imparted with both amplitude and phase noise picked up during propagation through the optical network. 'Imprinting' this noise into the Slave laser (to be used, e.g., as a Local Oscillator in coherent detection) is obviously not desirable. The same applies for phase regenerators (e.g., based on Phase Sensitive Amplification [9]), which rely on phase synchronization of the signal and the locally-generated phase-synchronized pumps without imprinting the noise on to the pumps. In these applications, the key property can be inferred from Eq. (1): This equation suggests that the OIL process acts as a low-pass optical filter (which, however, filters the signal phase only) whose bandwidth can be controlled by the OIL ratio $\rho_{O I L}$.

a)

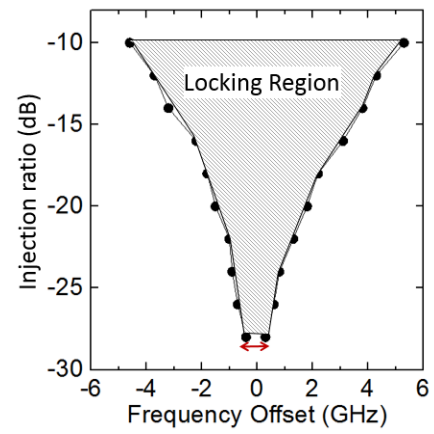

b)

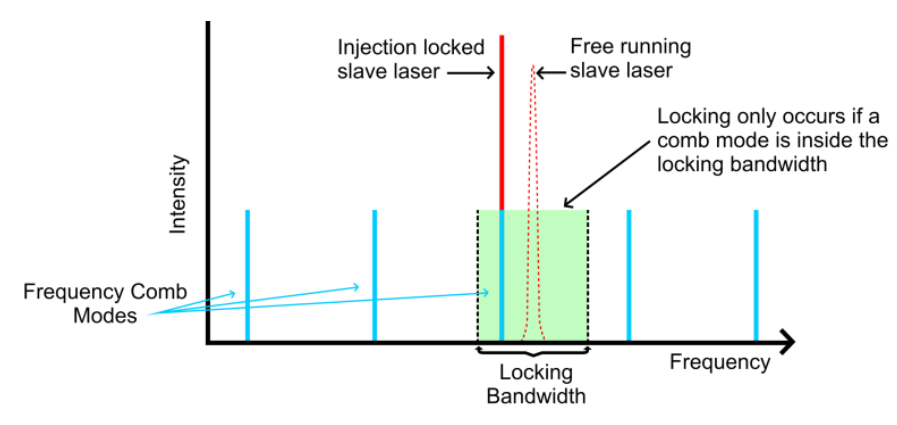

Fig. 1 (a) An example of low OIL ratio locking region. (b) OIL to a tone of an Optical Frequency Comb.2.3 Ultra-low OIL ratio

In some applications, it is desirable to get a very low $\mathrm{BW}_{O I L}$ with values of $\rho_{O I L}$ down to $-60 \mathrm{~dB}$ or even smaller [10]. An example is single tone extraction of an Optical Frequency Comb with a narrow tone spacing, e.g., 250 $\mathrm{MHz}$ [10], as commonly used in Metrology [11]. For such a narrow spacing, $\mathrm{BW}_{\text {OIL }}$ must be correspondingly small. 
At the same time, extracting a single comb tone necessarily involves very low power (as there are many tones when using such a narrow spacing), making OIL an ideal tool for such extraction purposes. In [12], up to 5 Slave lasers were locked to different comb tones, allowing for the generation of customized pulse shapes at $100 \mathrm{GHz}$ repetition rate. In [13], the ultra-low OIL ratio was used to re-amplify a CW signal carrying ultra-precise frequency information over a bi-directionally-operated optical network with high (>50 dB) gain.

\section{Carrier extraction with OIL}

To extract a signal carrier using OIL, the carrier either needs to be present in the signal (strongly-suppressed, but still present, e.g., [14], Fig. 2a), or the modulation needs to be stripped off all-optically first (an example is given in Fig. 2a [9]; another method is described, e.g., in [15]). In all these methods, however, the low-phase noise filtering property of OIL is the key, as it suppresses the data (e.g., Fig. 2a), or removes most of the noise (the portion of the noise that lies beyond the locking bandwidth) accumulated during signal propagation, which is key, e.g., in optical regeneration [9].

a)

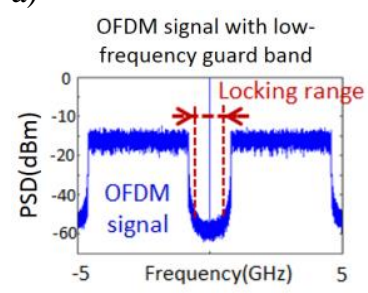

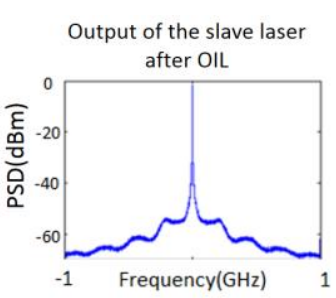

b)
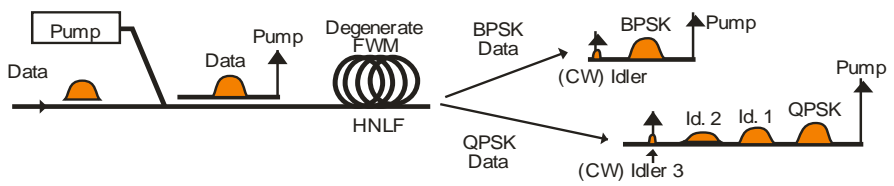

Fig. 2 (a) OFDM signal with low-frequency sub-carriers removed and a small pilot tone inserted. After OIL, the data signal is suppressed and the pilot tone phase-coherently amplified. (b) Modulation stripping - principle shown on an example of BPSK [9] and QPSK modulation.

\section{Conclusions}

Optical injection locking is an extremely simple technique with wide capabilities. It has several key properties and regimes of operation. Here, we review the key properties and opportunities for optical injection locking relevant to signal carrier extraction.

This research has received funding from EPSRC Fellowship Grant Agreement No. EP/K003038/1 and EPSRC grant EP/I061196X (The Photonic Hyperhighway).

\section{References}

1. A. Tartaglia, Optics For 5G: How can we combine low cost with demanding requirements?, ECOC2016, pp.239-250, Dusseldorf, 2016

2. R.J. Steed et al, Monolithically integrated heterodyne optical phase-lock loop with RF XOR phase detector, Opt. Express 19 (21) pp. 20048 20053 (2011).

3. F. Mogensen, H. Olesen, and G. Jacobsen, Locking conditions and stability properties for a semiconductor laser with external light injection, J. Qantum Electron QE-21 (7), pp. 784-703 (1985).

4. S.H. Lee et al, Bandwidth enhancement of injection-locked distributed reflector laser with wire like active regions, Opt. Express 18 (16), pp. 16370-16378 (2010).

5. H. Tsuchida, Tunable, narrow-linewidth output from an injection-locked high-power AlGaAs laser diode array, Opt. Lett. 19 pp. $1741-1743$ (1994).

6. Z. Liu, S. Farwell, M. Wale, D.J. Richardson, and R. Slavík, InP-based optical comb-locked tunable transmitter, OFC2016, paper Tu2K.2, 2016

7. A. Fragkos, A. Bogris, D. Syvridis, and R. Phelan, Amplitude noise limiting amplifier for phase encoded signals using injection locking in semiconductor lasers, J. of Lightwave Technol. 30 (5), pp. 764-771 (2012).

8. Y. Lai, H.A. Haus, and Y. Yamamoto, Squeezed vacuum from aplitude squeezed states, Opt. Lett 16 (19), pp. 1517-1519 (1991).

9. R. Slavík et al, Coherent all-optical phase and amplitude regenerator of binary phase-encoded signals, J. of Sel. Topics in Quantum Electron. 18 (2), pp. 859-869 (2012).

10. D.S. Wu, R. Slavík, G. Marra, and D.J. Richardson, Direct selection and amplification of individual narrowly spaced optical comb modes via injection locking: design and characterization, J. of Lightwave Technol., 31 (14), pp. 2287-2295 (2013).

11. http://www.menlosystems.com/en/products/optical-frequency-combs/fc1500-250-wg/

12. D.S. Wu, D.J. Richardson, and R. Slavík, Optical Fourier synthesis of high-repetition-rate pulses, Optica 2 (1), pp. 18-26 (2015).

13. J. Kim, H. Schnatz, D.S. Wu, G. Marra, D.J. Richardson, and R. Slavík, Optical injection locking-based amplification in phase-coherent transfer of optical frequencies, Opt. Lett 40 (18) pp. 4198-4201 (2015).

14. Z. Liu, J.Kim, D.S. Wu, D.J. Ricahrdson, and R. Slavik, Homodyne OFDM with Optical Injection Locking for Carrier Recovery, J. of Lightwave Technol 33 (1) (2015)

15. R. Slavík, J. Kakande, and D. J. Richardson, Feed-forward true carrier extraction of high baud rate phase shift keyed signals using photonic modulation stripping and low-bandwidth electronics, Opt. Express 19, 26594-26599 (2011). 